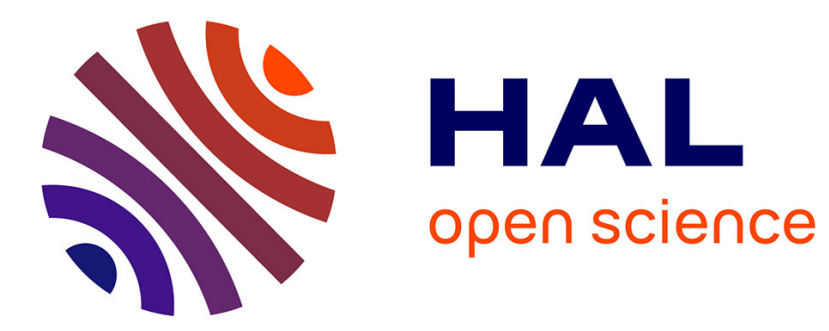

\title{
Le travail étiologique sous contrainte nationale Jean-Noël Jouzel
}

\section{To cite this version:}

Jean-Noël Jouzel. Le travail étiologique sous contrainte nationale: Les usages de la science dans les mobilisations contre les toxiques professionnels, des Etats-Unis vers la France. La Revue de l'IRES, 2012, 3 (74), pp.103 - 126. hal-02163416

\section{HAL Id: hal-02163416 \\ https://hal-sciencespo.archives-ouvertes.fr/hal-02163416}

Submitted on 24 Jun 2019

HAL is a multi-disciplinary open access archive for the deposit and dissemination of scientific research documents, whether they are published or not. The documents may come from teaching and research institutions in France or abroad, or from public or private research centers.
L'archive ouverte pluridisciplinaire HAL, est destinée au dépôt et à la diffusion de documents scientifiques de niveau recherche, publiés ou non, émanant des établissements d'enseignement et de recherche français ou étrangers, des laboratoires publics ou privés. 


\title{
Le travail étiologique sous contrainte nationale Les usages de la science dans les mobilisations syndicales contre les toxiques professionnels, des Etats-Unis vers la France
}

\author{
Jean-Nö̈l JOUZEL ${ }^{1}$
}

\begin{abstract}
Survenue au cœur des années 1990, la crise de l'amiante a marqué une rupture dans l'histoire des mobilisations de travailleurs en faveur de la reconnaissance des maladies professionnelles. Si la lutte contre les poisons industriels a constitué un enjeu politique important dans la construction du syndicalisme français à la fin du XIX ${ }^{\mathrm{e}}$ siècle (Gordon, 1993 ; Devinck, 2008), elle est devenue un objet de mobilisation nettement plus discret au cours du siècle suivant (Rosental, Omnès, 2009), même si plusieurs luttes sociales notables ont eu pour enjeu la dénonciation des dangers de certaines substances toxiques pour les travailleurs, sans que les syndicats y aient nécessairement tenu un rôle central (Pitti, 2010). L'émergence d'une mobilisation très visible publiquement dénonçant les dangers de l'amiante pour la santé des travailleurs voici près de 20 ans a donc contribué à réactiver ce type de conflits du travail. Par-delà l'histoire singulière de l'amiante, elle a eu pour suite d'autres mobilisations dénonçant les dangers de produits toxiques inhalés ou manipulés par les travailleurs : nanoparticules, éthers de glycol, perchloroéthylène...

La mobilisation syndicale contre l'amiante diffère néanmoins sensiblement de celles qui l'ont suivie contre d'autres toxiques professionnels, en raison du faible degré d'incertitude caractérisant la question des liens entre exposition à l'amiante et santé des travailleurs. Les dangers de cette fibre sont connus de longue date, et sa cancérogénécité est établie depuis le milieu des années 1970 (Chateauraynaud, Torny, 1999 ; Henry, 2007). La dénonciation des dangers de l'amiante pour les salariés a été facilitée par
\end{abstract}

1. Centre de sociologie des organisations, CNRS-Sciences Po. 
le fait que ce minéral entraîne une pathologie relativement rare, le mésothéliome, qui "signe » l'exposition du travailleur qui en est victime. Or, cette relation causale relativement claire fait de l'amiante une exception dans l'ensemble des toxiques professionnels, dont les effets pathogènes sont rarement spécifiques, et peuvent avoir de tout autres causes : hérédité, habitudes de vie, hasard. La concurrence étiologique entre une variété de causes possibles de la maladie rend alors l'imputation de cette dernière à l'environnement de travail et aux substances toxiques qui s'y trouvent autrement problématique et incertaine que dans le cas de l'amiante. La reconnaissance des maladies professionnelles induites par ces substances toxiques passe donc par la production de données venant étayer l'hypothèse d'un lien causal entre les premières et les secondes. Plusieurs travaux de sciences sociales, situés au croisement des science studies et de la sociologie des mouvements sociaux, ont déjà éclairé cette dimension des luttes sociales pour la reconnaissance des maladies causées par les expositions toxiques de travailleurs - mais aussi de riverains ou de consommateurs que nous proposons ici d'appeler travail étiologique. Ces travaux recourent notamment au terme d'« épidémiologie populaire » (Brown, 1987) pour décrire l'appropriation et l'utilisation d'outils et de concepts venus de l'épidémiologie académique (statistiques, cartographie...) par des " profanes » (Wynne, 1992) conduisant des " enquêtes » (Akrich et al., 2010) afin de rendre visibles des liens causaux entre substances toxiques et santé. Plusieurs d'entre eux soulignent combien le succès de ces tentatives passe bien souvent par l'enrôlement d'un ou de plusieurs professionnels de la recherche au service de la cause (Brown, 1987 ; Calvez, 2009). L'ambition de cet article est d'interroger la manière dont les organisations syndicales françaises recourent à des pratiques d'épidémiologie populaire pour légitimer la reconnaissance de liens causaux incertains entre divers toxiques professionnels et pathologies affectant les travailleurs.

Pour cela, nous nous proposons d'étudier le cas de la mobilisation des organisations syndicales françaises contre les éthers de glycol. Ces substances sont des solvants, synthétisés depuis les années 1930. Elles se répartissent en deux séries, $\mathrm{E}$ et $\mathrm{P}$, selon qu'elles sont dérivées de l'éthylène ou du propylène. Leur usage s'est principalement diffusé à partir des années 1960 et de la mise au point des peintures dites «à l'eau », dans la composition desquelles elles entrent en raison de leur capacité à être solubles à la fois dans l'eau et dans les corps gras. Les éthers de glycol ont par la suite été utilisés pour la fabrication de nombreux produits de consommation courante : encres, peintures, vernis, produits de nettoyage... Plusieurs centaines de milliers de travailleurs y sont exposés en France. Or, à la fin des années 1970, une équipe de toxicologues japonais dirigée par le professeur Nagano montra chez des rongeurs de laboratoire la toxicité pour 
la reproduction (stérilité et malformations intra-utérines) de certains éthers de glycol de série E, de loin les plus utilisés car les moins coûteux à synthétiser. Ces découvertes furent indirectement à l'origine d'une mobilisation d'organisations de travailleurs françaises contre l'usage des éthers de glycol à partir de la fin des années 1990. Cette mobilisation est d'autant plus intéressante à étudier qu'elle procède, pour partie, d'une dynamique d'importation de controverses alors en cours aux Etats-Unis autour des maladies professionnelles affectant les travailleurs des salles blanches de l'industrie des semi-conducteurs, dans lesquelles sont utilisées de nombreuses substances chimiques dont les éthers de glycol. L'étude de cette importation permet de rendre compte des singularités du travail étiologique produit par les organisations syndicales françaises pour dénoncer les éthers de glycol.

Cet article retrace la «carrière publique » (Crespin, 2006) des éthers de glycol comme enjeu de santé au travail de part et d'autre de l'Atlantique. Il repose sur une soixantaine d'entretiens réalisés avec les acteurs ayant pris part aux controverses autour des éthers de glycol aux EtatsUnis et en France, ainsi que sur une série d'observations du "Collectif éthers de glycol », qui a rassemblé à partir de l'an 2000 organisations syndicales, mutuelles et associations de victimes dénonçant la dangerosité de ces molécules pour les travailleurs. La première partie retrace l'histoire politique des luttes pour la reconnaissance des maladies des salles blanches aux Etats-Unis et rend compte de la part, relativement modeste, qu'y tinrent les éthers de glycol (I). La deuxième partie de l'article s'intéresse à l'importation de ces controverses en France à la fin des années 1990, et montre comment elle procède d'une dynamique de réduction étiologique aux seuls éthers de glycol d'un ensemble de pathologies potentiellement imputables à de multiples substances toxiques présentes dans les salles blanches (II). La troisième partie analyse les raisons de cette réduction ainsi que les difficultés rencontrées par le Collectif éthers de glycol pour construire une cause politique durable autour de ces substances (III).

\section{Les salles blanches en procès : le travail étiologique orienté par le droit}

Aux Etats-Unis, la mise en cause des éthers de glycol comme possible risque pour les travailleurs exposés a eu lieu à partir des années 1980 sous l'impulsion d'un cabinet d'avocats engagé dans un intense conflit judiciaire avec les firmes de l'industrie des semi-conducteurs, fortement consommatrices des ces substances. Mais dès le milieu des années 1990, à la suite de la substitution des éthers de glycol de série E par ceux de la série P dans cette industrie, ces avocats ont poursuivi leur dénonciation en mettant en avant les dangers de nombreuses autres molécules présentes dans les « salles 
blanches » où sont fabriquées les puces électroniques. Ils se sont pour cela appuyés sur le travail d'un scientifique critique vis-à-vis des risques professionnels dans ce secteur (I.1). Ils ont alors cherché à démontrer la volonté d'IBM de ne pas diffuser de données sur les maladies des travailleurs des salles blanches, donc à engager sa responsabilité dans la méconnaissance des liens entre travail et santé (I.2).

\section{I.1. D'un " effet salle blanche » à l'autre}

Le droit américain prévoit deux modalités principales d'indemnisation pour les travailleurs victimes de dommages corporels liés à leur travail. La première est assurantielle, et passe par les dispositifs de workers compensation dont l'ensemble des états fédérés se sont dotés à partir du début du $\mathrm{XX}^{\mathrm{e}}$ siècle. Pour obtenir une compensation, les travailleurs doivent alors démontrer le lien de causalité entre leurs pathologies et leur travail, mais sont exemptés de la démonstration d'une quelconque responsabilité de leur employeur. Cette voie de recours (dont le statut public ou privé varie selon les états) ne permet néanmoins que la prise en charge de maladies dont le lien de causalité avec le travail est relativement simple à établir. La deuxième voie d'indemnisation est le recours aux tribunaux de droit commun. Cette voie impose au travailleur de démontrer non seulement l'existence d'un lien de causalité entre son état de santé et son travail, mais également la responsabilité d'un tiers. A partir de la multiplication à la fin des années 1960 des procès intentés par des travailleurs atteints de pathologies chroniques liées à l'amiante, le recours à cette voie d'indemnisation est devenu de plus en plus massif dans le cas des maladies induites par l'exposition professionnelle à des substances toxiques (Ashford, Caldart, 1996). Ce mouvement a contribué à l'émergence d'un domaine spécifique de litiges, les toxic torts, réunissant les procès intentés par des consommateurs, des riverains ou des travailleurs s'estimant victimes d'intoxications liées aux produits que les industriels commercialisent, rejettent ou utilisent (Jasanoff, 1995). En règle générale, les travailleurs recourant à ce type de procédure ne poursuivent pas leur employeur, mais plutôt un ou plusieurs fournisseurs de substances chimiques toxiques. L'histoire politique des éthers de glycol aux Etats-Unis est liée à un procès singulier qui a opposé des travailleurs de l'industrie de semi-conducteurs au leader et principal employeur de ce secteur : la firme IBM.

La première controverse liée aux effets des éthers de glycol sur les travailleurs s'est déroulée en Californie, et plus précisément dans la Silicon Valley, où sont concentrées, au sud de la baie de San Francisco, les principales firmes du secteur des semi-conducteurs. Cette industrie consomme un grand nombre de substances de synthèse, à des doses relativement faibles, pour protéger les circuits imprimés sur les puces contre les impuretés. Au 
début des années 1980, les éthers de glycol de série E y sont utilisés dans un mélange photorésistant empêchant que la lumière provoque des courtscircuits sur la surface des puces. Les industries de ce secteur bénéficient alors d'une image relativement "propre ", notamment en raison du faible nombre d'accidents du travail y étant enregistrés chaque année. Mais dès la fin des années 1970, des travailleurs de ces firmes ainsi que certains de leurs riverains résidant dans la Silicon Valley se mobilisent pour dénoncer la toxicité des produits utilisés et rejetés par cette industrie. Cette dernière étant très peu syndicalisée, c'est principalement un cabinet d'avocats local - Alexander, Hawes and Audet - qui organise la contestation, en cherchant à la porter à la fois dans l'espace public et devant les tribunaux.

A partir du milieu des années 1980, les avocats du cabinet Alexander s'appuient sur des plaintes émises par des travailleuses des salles blanches ayant subi plusieurs fausses couches pour évoquer l'hypothèse d'un " effet salle blanche » sur le taux d'avortements spontanés des salariées de cette industrie, en lien avec les produits auxquels elles étaient exposées. En relayant publiquement ces inquiétudes, ils obtiennent des industriels qu'ils financent collectivement une vaste enquête épidémiologique destinée à vérifier la validité de l'hypothèse de l'effet salle blanche et à en identifier la cause. IBM, principale firme du secteur, réalise de son côté sa propre étude sur ses salariés. En 1992, ces deux enquêtes livrent leurs résultats. Elles confirment l'existence d'un effet salle blanche sur le taux de fausses couches subies par la main-d'œuvre féminine de cette industrie et en identifient une cause probable : les éthers de glycol de série E utilisés dans les procédés de photolithographie, bien que les travailleurs des salles blanches y aient été exposés à des doses inférieures aux valeurs limites réglementaires censées être protectrices. Au cours des deux années qui suivent, l'ensemble des industriels du secteur retirent les éthers de glycol de série E des salles blanches, pour les remplacer par des substances de série P.

Cette décision ne met pas un terme à la controverse. A partir de 1996, le cabinet Alexander rassemble un ensemble de plaintes de travailleurs des salles blanches souffrant de pathologies chroniques imputables selon eux aux produits qu'ils ont manipulés et inhalés sur leur lieu de travail. Le premier de ces plaignants est un salarié de l'usine IBM d'East Fishkill dans l'état de New York, atteint d'un cancer du testicule, et ayant constaté que les membres de son équipe ont, pour beaucoup d'entre eux, rencontré des problèmes similaires en dépit de leur jeune âge :

« Il travaillait avec des outils destinés à appliquer le mélange photorésistant sur les puces, et il avait 36 ans. [...] Quand il s'est renseigné, il a vu que des choses similaires étaient arrivées à d'autres personnes qui travaillaient avec lui. Il a trouvé un autre homme, membre de son équipe de 10 personnes, qui avait également un cancer du testicule. Quelles sont les 
probabilités pour que cela arrive par hasard ? Très basses. Cette équipe était formée de gens assez jeunes, et il y avait deux femmes avec des tumeurs du cerveau, une femme qui est morte d'un cancer du colon, deux hommes avec un cancer du testicule, et un homme souffrant d'un lymphome. Tout le monde était malade. Le plus vieux d'entre eux avait 36 ans. C'était très anormal ${ }^{2}$.»

A la suite de cette plainte, le cabinet Alexander se met en quête d'autres cas de travailleurs des salles blanches atteints de pathologies chroniques et acceptant de porter plainte contre IBM. En deux ans, il rassemble plus de 200 plaintes, en provenance de l'usine d'East Fishkill ou de celle de San Jose, dans la Silicon Valley. Ces plaintes sont liées à un ensemble de pathologies hétérogènes : cancers, malformations intra-utérines, stérilité... Il n'existe pas de données permettant d'imputer causalement cet ensemble de maladies à l'exposition à une substance spécifique. Ainsi, si les cas de stérilité et de malformations peuvent éventuellement être liés aux éthers de glycol, il n'existe pas alors de données toxicologiques sur la cancérogénécité de ces molécules. Pour exploiter le grand nombre de cas recueillis et obtenir une indemnisation de leurs clients, les avocats du cabinet Alexander ne cherchent pas à démontrer que l'exposition à telle ou telle substance induit tel ou tel type d'effets, mais bien que le travail en salle blanche, et les multiples expositions à de faibles doses de substances chimiques qu'il induit, est en lui-même toxique et génère un nombre anormalement élevé de pathologies chroniques. Pour étayer cette hypothèse, les avocats s'appuient sur les travaux d'un hygiéniste industriel de l'université de San Francisco, Joseph LaDou, engagé de longue date dans la dénonciation des dangers de l'industrie de semi-conducteurs. Selon ce chercheur, les travailleurs de ce secteur sont exposés à un risque de développer des pathologies chroniques variées non à cause de substances toxiques particulières, mais à cause du cocktail formé par la coexposition à une grande variété de substances toxiques présentes à faibles doses dans les salles blanches :

«Vous savez comment marchent les salles blanches ? L'air recircule en permanence pour protéger les puces des impuretés, et de très nombreux produits chimiques sont présents, en nombre beaucoup plus élevé que dans la plupart des process industriels. Plus l'industrie a évolué vers la fabrication de petites puces, de plus en plus petites, plus elle a eu besoin de ce process, et d'un grand nombre de produits. [...] Les salles blanches sont un environnement propice à des expositions croisées, à plusieurs produits, ou à de nouveaux produits créés par l'interaction des produits chimiques qui sont dans l'air que respirent les travailleurs. Mais que sait-on sur les

2. Entretien de l'auteur avec une avocate du cabinet Alexander, septembre 2004. 
expositions multiples ? Rien, ou presque rien. On n’a jamais réfléchi, et encore de manière très lacunaire, que sur des produits individuels ${ }^{3}$. »

\section{I.2. De l'ignorance comme ressource stratégique}

Cette stratégie judiciaire contraint les avocats à attaquer l'employeur lui-même, et non une ou plusieurs entreprises lui fournissant un ou des produits spécifiques. Pour démontrer la responsabilité d'IBM dans les souffrances de leurs clients, ils s'appuient sur un article publié en 1996 par quatre épidémiologistes de l'université d'Alabama dans la revue Epidemio$\log y$ sur les tumeurs du cerveau parmi les travailleurs des salles blanches ${ }^{4}$. L'article, reposant sur une enquête financée par IBM en 1985, concluait à l'absence de relation causale entre tumeurs du cerveau et travail en salle blanche. En revanche, il mentionnait l'existence d'un « fichier de mortalité » tenu par IBM depuis 1969, dans lequel étaient recensés les décès des anciens employés de la firme et leur cause probable. En utilisant cette information, le cabinet Alexander cherche à démontrer qu'IBM masque volontairement la dangerosité de ses produits pour les travailleurs. Les avocats exigent que la firme leur communique ce dossier, et utilisent la fin de non-recevoir qui leur est opposée comme un indice de la volonté d'IBM de cacher ce qu'elle sait des maladies des salles blanches.

Cette option stratégique permet aux avocats de faire de l'absence de données sur les maladies des travailleurs des salles blanches un argument légitimant la tenue d'un procès au cours duquel le juge pourra sommer IBM d'ouvrir à l'expertise judiciaire son fichier de mortalité. Pour arguer de sa bonne foi, la firme rappelle qu'elle a financé dix ans plus tôt une étude démontrant la dangerosité des éthers de glycol et a en conséquence retiré ces substances de ses salles blanches dès 1994. Les avocats du cabinet Alexander s'appuient à nouveau sur Joseph LaDou pour discréditer cette ligne de défense : selon le chercheur, l'enquête épidémiologique sur l'effet salle blanche, sous couvert de protection des travailleurs, a été modelée en conformité aux intérêts des industriels l'ayant financée. La possibilité de remplacer les éthers de glycol de série $\mathrm{E}$ par ceux de la série $\mathrm{P}$ a en effet selon lui permis de "résoudre » à moindre frais le problème des salles blanches sans investiguer davantage la piste de la toxicité du travail dans cet environnement chargé en particules chimiques diverses :

"[L'industrie] a utilisé cette recherche pour cibler les éthers de glycol comme la première cause des fausses couches. Cette appréciation exaspère le docteur Joseph LaDou [...] : "L'industrie a fait une étude étroite sur une seule question : les fausses couches, nous a-t-il dit. Cette étude n'a jamais prouvé qu'une seule substance était cause du problème, et ils n'ont jamais

3. Entretien de l'auteur avec Joseph LaDou, septembre 2004.

4. C. Beall et al., "Brain tumors among electronics industry workers », Epidemiology, vol. 7, $\mathrm{n}^{\circ} 2$, 1996, p. 125-130. 
essayé de revenir sur ces résultats pour démontrer que le problème avait été résolu par le retrait de cette substance. La question est beaucoup plus large que ce qu'ils veulent nous faire croire ${ }^{5}$." ’

Le procès intenté par le cabinet Alexander à IBM se déroule lors de l'hiver 2003-2004 à San Jose, et se solde par la défaite des plaignants (Jouzel, 2013). Il n’entre pas dans le propos de cet article de détailler cette histoire judicaire. Mais ce bref récit de la carrière publique des éthers de glycol comme enjeu de santé au travail outre-Atlantique nous donne à voir combien le droit oriente le travail étiologique effectué par les acteurs qui entendent dénoncer les dangers des toxiques professionnels pour la santé des travailleurs. Pour obtenir la reconnaissance des maladies affectant les travailleurs des salles blanches, les avocats ont été conduits à se désintéresser des éthers de glycol et à dénoncer les biais dans les études prouvant leur danger pour la main-d'œuvre en âge de procréer. Il leur a fallu à l'inverse construire, avec l'appui d'un scientifique, une étiologie beaucoup plus large des pathologies des travailleurs des salles blanches. Cette étiologie était difficile à étayer au vu des données toxicologiques et épidémiologiques disponibles. Mais, en dépit de leur échec final, le tour de force des avocats du cabinet Alexander fut de construire cette situation d'ignorance comme le produit d'une stratégie délibérée de dissimulation déployée par IBM ${ }^{6}$.

\section{Les syndicats français face à l'incertitude : comment dénoncer les dangers des éthers de glycol ?}

En France, les éthers de glycol ne sont traités publiquement comme un possible enjeu de santé au travail qu’à partir du milieu des années 1990, à l'occasion du licenciement par l'INRS d'un de ses chercheurs travaillant spécifiquement sur la dangerosité de ces molécules. Une faible mobilisation syndicale empêche alors toutefois cette controverse d'émerger durablement (II.1). Les avocats américains du cabinet Alexander vont contribuer à réactiver la mobilisation contre les éthers de glycol au début des années 2000, via le site IBM de Corbeil-Essonnes. S’ils privilégient désormais une analyse plurifactorielle de l'exposition aux produits toxiques dans les salles blanches, ils s'appuient malgré tout sur le début d'action collective qui a pris forme autour des éthers de glycol. Cette mobilisation prend une

5. San Francisco Bay Guardian, hebdomadaire gratuit d'information locale dans la baie de San Francisco, 26 avril 2000.

6. Nombre de travaux contemporains de sciences sociales sur « la production sociale de l'ignorance " montrent que cette dernière constitue une ressource stratégique pour les firmes qui souhaitent dissimuler la nocivité des produits qu'elles utilisent, qu'elles commercialisent ou qu'elles rejettent dans l'environnement (Markowitz, Rosner, 2003 ; Oreskes, Conway, 2010 ; Proctor, 2012). L'histoire du procès des salles blanches aux Etats-Únis montre à l'inverse une situation dans laquelle ce sont les travailleurs plaignants et leurs avocats qui tirent profit de la méconnaissance des liens entre travail et santé, en en imputant la responsabilité à la firme. 
ampleur nationale et se politise mais reste centrée sur la seule question des éthers de glycol (II.2).

\section{II.1. La difficile dénonciation syndicale des éthers de glycol}

La carrière publique des éthers de glycol comme enjeu de santé au travail en France a connu une trajectoire en pointillés, marquée par une succession de périodes de publicisation des conflits liés à ces molécules entrecoupées par des phases de retombée dans l'oubli médiatique et politique, sur une période d'une dizaine d'années, du milieu des années 1990 au milieu des années 2000. La publication des travaux de Nagano sur la reprotoxicité des ces substances à la fin des années 1970 ne déclenche aucune protestation contre l'usage industriel des éthers de glycol parmi les organisations de travailleurs en France. Le seul acteur s'intéressant à ces molécules est l'Institut national de recherche et de sécurité (INRS). Cet organisme de recherche dédié aux questions de santé au travail a un statut d'association relevant de la loi de 1901, et est géré depuis la fin des années 1960 de façon paritaire par les syndicats patronaux et de travailleurs bénéficiant d'une présomption de représentativité (CGT, CFDT, Force ouvrière, Confédération générale des cadres, Confédération française des travailleurs chrétiens). Ce mode de gouvernance est cohérent avec l'orientation générale des politiques de santé au travail, qui reposent historiquement sur une "gestion négociée » (Courtet, Gollac, 2012) des choix relatifs à la prévention et à la reconnaissance des maladies professionnelles, associant fortement organisations syndicales et patronales.

En 1987, l'INRS charge un de ses chimistes et toxicologues, André Cicolella, de diriger un programme de recherche conjoint avec l'Inserm sur ces substances et leurs dangers pour l'homme. En avril 1994, au moment où s'ouvre à Pont-à-Mousson le symposium international de synthèse qu'il a organisé sur cette question, cet ingénieur est licencié par sa direction pour insubordination. Ce licenciement, jugé abusif par la cour d'appel en 1998, fait suite à des conflits entre les membres des équipes Inserm et INRS travaillant sur le projet éthers de glycol. Ce conflit du travail est à l'origine du premier épisode de mobilisation en lien avec les effets des éthers de glycol sur les travailleurs (Jouzel, 2007), et ce au moment-même où les acteurs américains de la controverse de l'effet salle blanche cessent de s'intéresser à ces substances. Il donne notamment lieu à des manifestations de soutien au chercheur licencié de la part de spécialistes de santé au travail, d'inspecteurs du travail ou de responsables syndicaux. Ces acteurs dénoncent le licenciement comme résultant de l'influence du patronat sur l'INRS et de la volonté de l'industrie chimique d'interrompre un projet de recherche compromettant pour les éthers de glycol. Cette mobilisation fait cependant long feu, notamment en raison de la part modeste qu'y tiennent les 
organisations syndicales. Ces dernières, associées à la gestion paritaire de l'INRS, peinent à participer à une action dénonçant le manque d'indépendance de cette institution, et contribuent ainsi à laisser les éthers de glycol retomber dans le silence :

«Allez voir [...] si vous voulez comprendre pourquoi une organisation paritaire comme l'INRS s'est comportée de cette façon-là, pourquoi les organisations syndicales ne se sont pas comportées comme elles auraient dû, pourquoi elles ont soutenu la direction de l'INRS. Y compris ma propre organisation syndicale, j'étais responsable CFDT, et la CFDT a soutenu le directeur de l'INRS. J'ai eu une lettre d'un des administrateurs, me disant : "Monsieur, ça ne change pas nos relations, etc." Alors qu'il y avait un enjeu de santé publique ${ }^{7}$.»

Les mois et les années qui suivent le licenciement d'André Cicolella sont marqués par le scandale de l'amiante. La publicisation de ce dernier est notamment alimentée par un fort engagement de deux organisations syndicales (la CGT et la CFDT) aux côtés des victimes de l'amiante (Henry, 2007). Cette mobilisation marque le retour de l'action syndicale à un registre de dénonciation critique des risques professionnels, alors que le $\mathrm{XX}^{\mathrm{e}}$ siècle a plutôt vu les logiques de négociation paritaire s'institutionnaliser fortement autour des enjeux de santé au travail. Dans ce contexte, les organisations syndicales les plus engagées dans la dénonciation de ce conflit cherchent à réactiver une mobilisation dénonçant les dangers des éthers de glycol au moment où le traitement médiatique du scandale de l'amiante tend à se «normaliser» (Henry, 2003), au cours de l'année 1997. La CGT et la CFDT, accompagnées par une association de victimes d'accidents du travail et de maladies professionnelles (la Fédération nationale des travailleurs et des handicapés - Fnath) et par une fédération de mutuelles (la Mutualité française) commencent alors à dénoncer publiquement les dangers des éthers de glycol, en se faisant le relais des alertes lancées par André Cicolella sur ce sujet. Ce collectif d'organisations exige l'interdiction des éthers de glycol de série $\mathrm{E}$ et leur substitution par ceux de la série P. Cet ensemble d'acteurs évoque le cas des éthers de glycol comme une possible « affaire de l'amiante-bis », contribuant à mettre en alerte les administrations de la santé au travail et de la santé publique (Jouzel, 2013). Cette mobilisation tourne cependant de nouveau court. Les ministères du Travail, de la Santé et de l'Environnement y répondent par la saisine d'une expertise collective de l'Inserm, dont les conclusions, rendues à l'automne 1999, restent très générales et ne proposent aucune mesure d'interdiction, se limitant à des recommandations relatives à l'information des travailleurs exposés et à l'approfondissement des connaissances. Ces conclusions contribuent à délégitimer l'idée qu'il existe un véritable problème de santé au travail lié

7. Entretien de l'auteur avec André Cicolella, novembre 2003. 
aux éthers de glycol, et à pacifier provisoirement les controverses autour de ces molécules.

\section{II.2. D'une usine à l'autre : l'importation ambiguë de l'affaire IBM}

Le troisième épisode de mobilisation d'organisations de travailleurs en lien avec les éthers de glycol a lieu à l'été 2000. Cette réactivation de la mobilisation est, pour partie, la conséquence des évolutions de l'affaire IBM aux Etats-Unis. A partir de 1998, les avocats du cabinet Alexander commencent en effet à s'intéresser à une usine IBM construite en France, à Corbeil-Essonnes, dans les années 1950, selon un plan identique à celui de l'usine d'East Fishkill d'où proviennent plusieurs dizaines de leurs clients. Ils approchent les délégués syndicaux CGT et CFDT de cette usine dans l'espoir d'obtenir des données sur d'éventuelles pathologies affectant les travailleurs de l'usine d'IBM Corbeil-Essonnes, afin de renforcer l'hypothèse de la toxicité du travail en salle blanche. Ces syndicalistes ont déjà cherché, en vain, à mobiliser les salariés du site autour de ce problème au cours des mois précédents, à la suite des prises de position de leurs confédérations respectives en faveur de l'interdiction des éthers de glycol. Un plan social lié à la revente de l'usine par IBM à sa filiale Altis est alors en cours, qui contribue à reléguer les enjeux de santé au travail au second plan des préoccupations des travailleurs.

A l'été 2000, les avocats du cabinet Alexander proposent aux syndicalistes du site, désormais passé sous le contrôle d'Altis, de réaliser un « appel à témoignages » auprès des travailleurs ayant œuvré en salle blanche du temps de la direction d'IBM et souffrant de pathologies chroniques. Les syndicalistes acceptent, avec le soutien de leurs confédérations. Le cabinet Alexander finance la rémunération d'un expert médical chargé d'accueillir et d'éventuellement examiner les «témoins », puis d'exploiter statistiquement les données recueillies, tandis que les syndicats organisent matériellement la permanence et l'accueil des travailleurs :

" Il faut savoir qu'Alexander est venu avec une enveloppe... Parce qu'en fait, bien souvent, on est confronté à des limites dans les recherches et dans l'action, qui sont liées aux problèmes financiers. Donc il a amené une enveloppe en disant: "Je suis prêt à mettre tant pour que vous fassiez cette étude." Ce n'est quand même pas une chose négligeable. De l'ordre de 100000 ou 150000 francs. Donc ça nous permet de recruter un expert qu'on paie, un toubib spécialisé, il est à la fois pédiatre de formation et médecin du travail, donc ça nous permet d'avoir quelqu'un. Ce n'est pas le tout d'envoyer un appel à témoignages, c'est que les gens vont venir, ils vont expliquer, donc en tant que médecin du travail, il y a une méthodologie aussi à respecter, pour essayer de repérer les expositions et les symptômes. 
Donc, vous voyez, ça ne peut pas être n'importe qui, un syndicaliste normal, qui peut traiter ce genre de choses $^{8}$. »

Pour les avocats californiens, le but de la manœuvre n'est pas spécifiquement de repérer des victimes des éthers de glycol. En 2000, le processus de marginalisation de ces substances dans le conflit autour des salles blanches de la Silicon Valley est achevé. C'est alors à d'autres toxiques professionnels et d'autres pathologies que les avocats des plaignants américains contre IBM s'intéressent. Pour eux, l'appel à témoins doit permettre de faire émerger, à Corbeil et East Fishkill, des cas de pathologies similaires, mais non nécessairement liées aux éthers de glycol. Ils n’ont cependant guère d'autres choix que de s'appuyer sur le début d'action collective qui a alors pris forme au sein de l'usine de Corbeil-Essonnes autour des éthers de glycol.

Les syndicalistes CGT et CFDT du site, ainsi que les organisations préalablement mobilisées dans la dénonciation des dangers des éthers de glycol, présentent quant à eux l'appel à témoignages comme un dispositif d'enquête visant spécifiquement à rendre visibles des cas de victimes de ces substances. Cette présentation est largement reprise par les médias, qui relatent massivement l'appel à témoignages en ne mentionnant que les éthers de glycol parmi les toxiques soupçonnés d'être nocifs pour la santé des travailleurs des salles blanches. Cette dynamique de focalisation sur ces seules substances s'étend rapidement à des acteurs du champ politique, notamment les Verts, qui publient le 9 août un communiqué de presse demandant « l'interdiction des éthers de glycol toxiques, la mise en place rapide d'un dépistage des salariés ayant été en contact avec cette substance, l'indemnisation des victimes [ainsi que] l'ouverture d'une enquête judiciaire afin d'établir la responsabilité à tous les niveaux de ceux qui savaient et ont laissé faire ${ }^{9}$ " .

Les organisations de travailleurs prolongent et amplifient cette dynamique de focalisation, tant au niveau local que national. Les délégués syndicaux de l'usine de Corbeil-Essonnes centrent ainsi leur mobilisation sur les seuls éthers de glycol. Ils obtiennent dès le 12 septembre 2000 de leur nouvelle direction la tenue d'une réunion du CHSCT entièrement consacrée aux « éthers de glycol sur le site IBM de Corbeil ». Le médecin du travail du site puis un expert en toxicologie y délivrent des points d'information relatifs à la seule question de la toxicité de ces molécules et de leur utilisation sur le site. Les nombreuses autres substances présentes dans les salles blanches et dénoncées comme dangereuses par les avocats du cabinet Alexander ne sont pas évoquées au cours de la réunion. Les syndicalistes présents soulignent la responsabilité d'IBM dans l'« exposition du personnel aux éthers de glycol », exigent des informations sur les tonnages

8. Entretien de l'auteur avec le représentant de la CGT au sein du Collectif éthers de glycol, juin 2003. 9. Communiqué de presse des Verts, 9 août 2000. 
utilisés chaque année sur le site, etc. ${ }^{10}$. Mais ils n’abordent pas la question des autres produits mis en cause par les avocats américains. Le déroulement de la réunion du CHSCT, quoiqu'évidemment conflictuel, repose sur une définition partagée du problème des salles blanches, réduit aux seules expositions à ces molécules.

Les organisations confédérales de ces syndicalistes adoptent ce même point de vue sur le problème des salles blanches. Sitôt l'appel à témoignages lancé, la CGT et la CFDT nourrissent la dynamique de focalisation médiatique sur les seuls éthers de glycol. Elles s'efforcent de présenter publiquement l'appel à témoignages comme une tentative de recherche de victimes de ces substances, et non de celles des cocktails de molécules présentes dans les salles blanches. Ces organisations parviennent à pérenniser le mouvement de protestation contre les éthers de glycol réactivé par l'appel à témoignages. A partir de celui-ci, elles donnent à leur coalition l'appellation de «Collectif éthers de glycol». Elles y sont rapidement rejointes par deux autres acteurs liés au monde de la santé au travail : le Syndicat national des professionnels de la santé au travail (SNPST ${ }^{11}$ ) et la Fédération des mutuelles de France $\left(\mathrm{FMF}^{12}\right)$. De plus, la Ligue contre le cancer s'associe aux activités de ce collectif. Cette dénomination signale la volonté des organisations syndicales et de leurs alliés de traiter les éthers de glycol comme des substances à part, caractérisées par une dangerosité singulièrement préoccupante. En 2003, ces associations officialisent cette appellation en signant une « charte du Collectif éthers de glycol» définissant les objectifs de la mobilisation : « 1 : faire interdire en milieu professionnel les éthers de glycol toxiques pour la reproduction », «2: faire réaliser une cartographie de la population concernée », « $3:$ susciter des recherches indépendantes sur les effets des éthers de glycol », « 4 : faire évoluer la réparation pour les victimes des éthers de glycol ». Les contours d'une mobilisation initialement lâche se trouvent ainsi progressivement institutionnalisés, contribuant à légitimer la spécificité de la question des éthers de glycol.

Ce processus d'importation de l'affaire IBM associé à une réduction aux seuls éthers de glycol des substances mises en cause par les organisations syndicales et leurs alliés nous conduit à poser trois séries de questions. La première a trait à l'étonnement que suscite une telle réduction étiologique. Pourquoi les organisations de travailleurs membres du Collectif ont-elles si activement encouragé une lecture politico-médiatique ramenant l'affaire IBM aux seuls dangers des éthers de glycol ? Pourquoi ont-elles effectué ce travail de réduction étiologique aux seuls éthers de glycol de l'effet salle blanche, à rebours de la dynamique d'élargissement initiée au

10. Compte rendu de la réunion du CHSCT d'Altis-Corbeil-Essonnes, 12 septembre 2000.

11. L'actuel SNPST était jusqu'au début des années 2000 le Syndicat national des professionnels de la médecine du travail.

12. Créée en 1977, la FMF est devenue membre de la Mutualité française en 2002. 
même moment par les avocats californiens? La seconde porte sur l'usage de la science dans cette dynamique de réduction. Quel type d'arguments les organisations syndicales ont-elles mobilisés pour légitimer leur interprétation du sens de l'appel à témoignage ? En quoi l'appui cognitif fourni par André Cicolella aux organisations syndicales a-t-il favorisé cette réduction étiologique ? Enfin, une troisième série de questions porte sur les effets politiques de cette réduction sur le destin de la cause politique construite par les organisations syndicales des éthers de glycol. Celle-ci s'est-elle avérée durable dans le temps ? Comment s'est-elle articulée avec les autres dimensions de l'action syndicale en matière de prévention des maladies professionnelles, et notamment avec la participation aux instances de concertation qui gèrent les risques professionnels?

\section{L'épidémiologie populaire sous contrainte politique}

Les éthers de glycol étant substituables par d'autres produits, leur transformation en enjeu de santé publique semble politiquement acceptable, et ne conduit pas les organisations syndicales à devoir faire un arbitrage entre la santé des travailleurs et la défense de leur emploi (III.1). Pour autant, les preuves scientifiques de leur toxicité sont si controversées (III.2) que les acteurs administratifs et académiques n'appuient pas le Collectif éthers de glycol, reléguant cette question dans l'oubli (III.3).

\section{III.1. A la recherche d'une cause politique acceptable}

Comme l'ont souligné une série de travaux de sociologie politique, la constitution par les organisations syndicales d'une cause politique dont l'objet est la dénonciation publique des dangers d'un toxique professionnel est bien souvent rendue difficile en raison de ses possibles implications économiques. Dans un contexte de sous-emploi chronique, ce type de mobilisation peut en effet induire un risque d'interdiction de substances qui, quoique nocives, sont profitables pour des secteurs d'activité qu'il convient de ne pas mettre en péril. Les difficultés rencontrées par les organisations syndicales pour entrer dans les luttes sociales liées à l'amiante au cours des années 1970 et 1980 illustrent bien cette tension entre protection de la santé des travailleurs et défense de leur emploi (Chateauraynaud, Torny, 1999 ; Henry, 2007 ; Thébaud-Mony, 2007). Ces difficultés ont constitué un obstacle à la dénonciation syndicale des dangers des éthers de glycol. Les syndicalistes engagés dans le Collectif éthers de glycol ont pu être confrontés à d'importantes contestations de la part de travailleurs de leur base, inquiets des effets économiques d'une possible interdiction des éthers de glycol:

" Je me suis même fait agresser par un gars, qui m'a dit : "Mais vous êtes dégueulasses, vous demandez l'interdiction des éthers de glycol, et moi 
je fais quoi ? Je suis formulateur, je fais quoi pour remplacer mon éther de glycol ?" [...] C'était un gars qui était ingénieur chimiste donc, j’allais dire, ça n'était pas un gars inconscient des possibilités d'innovation ou des trucs comme ça. C'était un gars qui était vraiment dans le coup. [...] Ils ont d'abord râlé comme des veaux en disant : "Vous êtes complètement fous, vous interdisez les éthers de glycol, on va les remplacer par quoi ${ }^{13}$ ? " "

La cause politique de la dénonciation des éthers de glycol présentait à cet égard une vertu, que les syndicalistes membres du Collectif ont constamment mise en avant pour obtenir l'adhésion de leur base comme de leur hiérarchie : les éthers de glycol qu'ils pointaient comme dangereux pour les travailleurs pouvaient être remplacés par d'autres, qui ne l'étaient pas. Tout au long de son existence, le Collectif a insisté sur le fait que le problème des éthers de glycol pouvait être résolu, pour un faible coût économique, par la substitution des substances de série E, pour lesquelles existaient des données de reprotoxicité, par celles de la série P :

"Cette décision [linterdiction des éthers de glycol toxiques pour la reproduction en milieu professionnel] est d'autant plus facile à prendre que les produits de remplacement existent dans la famille des éthers de glycol et que cette substitution peut se faire sans difficulté technologique majeure. Un certain nombre d'entreprises ont déjà procédé à des changements et deux fabricants d'éthers de glycol (les sociétés Dow et Arco) ont arrêté la production des éthers de glycol toxiques pour la reproduction, en raison de cette toxicité ${ }^{14}$.»

En mettant en avant cette propriété du problème des éthers de glycol, le Collectif en a grandement neutralisé la charge critique. Il a accompagné, favorisé et, peut-être, accéléré le mouvement de substitution de la série $\mathrm{E}$ par la série P qui s'est structuré à partir du début des années 1990 dans la plupart des secteurs industriels des pays occidentaux. Alors qu'à la fin des années 1990, 17000 tonnes d'éthers de glycol de série E et 12500 de série P étaient utilisées en France, la proportion s'est inversée au cours de la décennie suivante, avec 13000 tonnes pour la série E et 19000 tonnes pour la série $\mathrm{P}$ en $2006^{15}$. Les quantités produites des quatre substances les plus notoirement reprotoxiques de la série $\mathrm{E}$ ne constituaient alors plus que $3 \%$ de ce qu'elles étaient en 1993. L'acuité du problème de santé au travail posé par l'exposition des salariés aux éthers de glycol a donc rapidement diminué. La mobilisation du Collectif n’a pas visé à faire interdire des substances représentant un danger public majeur, mais plutôt à constituer un précédent mobilisable pour de futures demandes d'interdiction de

13. Entretien de l'auteur avec un représentant de la CFDT au sein du Collectif éthers de glycol, juillet 2003.

14. Lettre adressée par la CGT, la CFDT, la Fnath et la Mutualité française à Martine Aubry, ministre de l'Emploi et de la solidarité, 20 avril 1998.

15. Afsset, "Ethers de glycol. Synthèse des connaissances sur les expositions de la population générale et professionnelle en France », Rapport d'expertise collective, février 2008. 
molécules sur la base d'essais sur l'animal et non de données épidémiologiques obtenues dans des conditions réelles d'exposition :

«Il faut bien remettre le problème des éthers de glycol dans son contexte. C'est-à-dire que c'est un tout petit problème de la santé au travail. C'est un problème qui relève de la chimie, des risques toxiques, sur une toute petite série de produits. Quand on interroge sur ce sujet un toxicologue, bon, les éthers de glycol, il vous dira : "Pour moi ce n'est pas le problème." Mais pour nous c'est le problème. C'est un problème politique. Parce que si on arrive sur ce petit dossier, avec notre collectif, avec le support des partenaires sociaux et des associations, à obtenir une interdiction symbolique, comme ça a été fait pour l'amiante, mais pour un produit chimique, cette fois-ci, on espère mettre le doigt dans l'engrenage d'un certain nombre d'autres interdictions ${ }^{16}$. »

\section{III.2. Construire un lien causal légitime}

Pour assurer la légitimité politique de sa cause, le Collectif éthers de glycol doit cependant produire des données étayant l'hypothèse de la dangerosité de ces substances pour les travailleurs. Or, les résultats de l'appel à témoignages auprès des salariés de l'ancienne usine IBM de CorbeilEssonnes sont à cet égard décevants. Loin de mettre en évidence de nombreux cas d'atteintes aux fonctions de reproduction susceptibles d'être causalement reliés à ces molécules, ils ne révèlent pour l'essentiel que des cas de cancers hétérogènes, parmi lesquels huit cas de cancers du testicule. La littérature disponible n'indique pas que les éthers de glycol puissent causer des cancers de ce type. Pourtant, le Collectif s'appuie sur l'expertise d'André Cicolella pour exploiter statistiquement cet agrégat de cancers et l'utiliser comme un argument pour étayer la thèse de la dangerosité des éthers de glycol pour les travailleurs.

André Cicolella et le Collectif réalisent tout d'abord une estimation statistique sur la base d'hypothèses relatives au nombre de travailleurs passés par les salles blanches à partir de 1975, date de début de l'utilisation des éthers de glycol par IBM, soit 4700 à 7800 travailleurs. Par comparaison avec la distribution attendue des cancers du testicule pour cette population, le chiffre de huit cas constitue selon André Cicolella un excédent statistique, neuf fois supérieur à la «normale». Si cette tentative d'épidémiologie populaire légitime l'idée que le travail dans les salles blanches d'IBM a été un facteur de maladies professionnelles, reste à démontrer que les éthers de glycol en ont été la cause. Pour cela, André Cicolella s'appuie sur une étude publiée en 1998 par des chercheurs américains du National Toxicology Program (NTP), qui a mis en évidence une augmentation des tumeurs du foie et de l'estomac chez les souris exposées à l'EGBE, un éther de glycol

16. Entretien avec un membre du Collectif éthers de glycol, novembre 2003. 
de série $\mathrm{E}$ très couramment employé, notamment dans les salles blanches, et des effets « équivoques » sur le foie des rats ${ }^{17}$. Ces données permettent selon l'ancien chercheur de l'INRS d'étayer l'hypothèse de mécanismes de cancérogénèse propres aux éthers de glycol et susceptibles d'expliquer les cas de cancers du testicule observés parmi les anciens travailleurs venus participer à l'appel à témoignages.

L'extrapolation à l'homme des données de cancérogénèse obtenues par le NTP fait cependant l'objet de controverses au début des années 2000. Aux Etats-Unis, l'Environmental Protection Agency refuse de classer l'EGBE comme agent cancérogène pour l'homme. En revanche, Santé Canada, agence en charge des politiques sanitaires canadiennes, propose, sur la base de cette même étude, une valeur-limite pour l'EGBE de $40 \mathrm{mg} / \mathrm{m}^{3}$. Le Centre international de recherche sur le cancer (Circ) tranche la question en 2004, en classant l'EGBE dans sa « catégorie 3 », regroupant les substances ne pouvant « être classées comme cancérogènes pour l'homme sur la base de preuves insuffisantes chez l'homme et insuffisantes ou limitées chez l'animal ». Le Circ s'appuie sur une étude parue en 2002 dans la revue Toxicology, qui impute au léchage du pelage la pénétration d'EGBE dans l'organisme du rongeur et se prononce contre une possible extrapolation à l'homme des résultats du NTP. En dépit de ces données, André Cicolella s'efforce de construire une argumentation favorable à l'hypothèse de la cancérogénécité des éthers de glycol. Il stigmatise notamment les auteurs de l'étude parue dans Toxicology comme des «scientifiques travaillant dans l'industrie chimique ", pour convaincre les membres du Collectif que les éthers de glycol peuvent être cancérogènes :

«Etude NTP, 1998 : cette étude menée sur le rat et la souris sur deux ans a montré que EGBE était cancérogène chez la souris, et, avec moins de force, chez le rat mâle [...]. Green (2002) montre que le métabolite acide de EGBE [...] est responsable d'une destruction des cellules, suivie d'une multiplication claire.

[...] Il y a discussion de la part des scientifiques travaillant dans l'industrie chimique sur l'extrapolation des résultats à l'homme.

[...] Cancers de l'estomac : la thèse de Green est que EGBE est présent dans l'estomac parce qu'il est ingéré. Comme les animaux se lèchent le poil, ce qui n'est pas le cas de l'homme, il n'y a pas de risque pour l'homme. Ce raisonnement est curieux, car le métabolite acide de EGBE est présent dans tous les compartiments, puisqu'il provient de la transformation de EGBE. S'il est démontré qu'il stimule la prolifération cellulaire, dans le

17. National Toxicology Program, « Toxicology and Carcinogenesis Studies of 2-Butoxyethanol (CAS n 111-76-2) in F344 Rats and B6C3F1 Mice Inhalation Studies », NTP TR-484 NIH Publication $n^{\circ} 98-3974,1998$. 
préestomac, on peut considérer qu'il en est potentiellement de même sur tous les autres sites où EGBE est métabolisé ${ }^{18}$. »

Sur la base de cette interprétation des données toxicologiques, André Cicolella conforte l'idée que les cas de cancers du testicule signalés lors de l'appel à témoignages peuvent être imputés aux éthers de glycol présents dans les salles blanches, et notamment à l'EGBE. Les organisations membres du Collectif relaient d'abord cette interprétation des résultats de l'appel à témoignages, en affirmant que les huit cas de cancers recensés à Corbeil-Essonnes constituent un agrégat statistiquement inattendu, similaire à celui observé dans l'usine d'East Fishkill, et imputable aux éthers de glycol présents dans les salles blanches :

«Si les effets sur la reproduction de certains éthers de glycol ne sont plus contestés, une interrogation s'est faite jour sur les effets cancérogènes, notamment à partir des résultats positifs de la première étude de toxicité chronique sur EGBE ainsi que des observations particulièrement troublantes de nombreux cas de cancer dans l'industrie des semi-conducteurs. Nous demandons [...] qu'un programme d'étude soit conduit pour répondre à cette grave interrogation. [...] De telles études ont été menées dans cette industrie qui ont montré un excès d'avortements spontanés et une hypofertilité. Il est temps, au vu des taux anormaux de certains cancers (neuf fois plus de cancers du testicule à l'usine de Corbeil-Essonnes comme à l'usine sœur d'East Fishkill aux Etats-Unis), de conduire une étude sous l'égide d'un organisme scientifique dont la rigueur est incontestable. Il vous appartient de le saisir ${ }^{19}$. »

\section{III.3. Une cause impossible}

En dépit des efforts déployés par le Collectif pour réactiver durablement une mobilisation centrée sur les effets des éthers de glycol sur la santé des travailleurs, cette cause politique retombe à nouveau, et, semble-t-il, durablement, dans l'oubli à partir du milieu des années 2000. Le Collectif éthers de glycol n'a jamais été officiellement dissout, mais il est aujourd'hui inactif, et sa dernière prise de position publique remonte à 2006. La principale raison de cette démobilisation réside dans l'incapacité du Collectif à intéresser durablement les acteurs des politiques de santé au travail à la cause des éthers de glycol. La gestion négociée des risques professionnels a pour conséquence que la reconnaissance des maladies du travail repose davantage sur l'état du rapport de force entre organisations patronales et syndicats de travailleurs que sur l'état d'avancée des connaissances scientifiques (Déplaude, 2003). Dans un contexte économique et industriel

18. André Cicolella, « Ethers de glycol. Etat de la réglementation et des connaissances scientifiques », document interne au Collectif éthers de glycol, février 2003.

19. Lettre du Collectif éthers de glycol à Elisabeth Guigou, ministre de l'Emploi et de la Solidarité, 30 mars 2001. 
difficile, et compte tenu des divisions entre organisations syndicales, ces dernières sont rarement en position de force dans ces négociations ${ }^{20}$. Elles ont donc un besoin constant de trouver des appuis auprès de l'Etat ou d'experts « neutres » pour faire peser les décisions en leur faveur en dépit de la capacité des industriels à mobiliser de puissantes ressources en expertise légitime (notamment des professeurs d'université praticiens hospitaliers) (Déplaude, 2003 ; Henry, 2011).

Or, le Collectif éthers de glycol peine à trouver ce type d'appui dans les années qui suivent l'appel à témoignages d'août 2000. Si le ministère du Travail, dans un contexte de sortie précaire de la crise de l'amiante, a contribué à légitimer le problème des éthers de glycol en commandant dès 1998 une expertise collective de l'Inserm sur ce sujet, il se montre par la suite beaucoup plus défiant vis-à-vis des positions du Collectif. Au sein du ministère, la direction des Relations du travail, en charge des questions de santé au travail, œuvre à proposer une approche élargie à l'ensemble des substances toxiques pour la reproduction des travailleurs exposés. A la suite des conclusions de l'expertise collective de l'Inserm, elle propose un ensemble de textes étendant aux substances reprotoxiques les restrictions d'usage en milieu professionnel des substances cancérogènes valant sur le sol de l'Union européenne. L'adoption, le $1^{\text {er }}$ février 2001, de ces décrets signe la victoire de cette direction ministérielle sur le cabinet du ministère du Travail, beaucoup plus favorable à des mesures spécifiquement centrées sur les éthers de glycol dans une logique d'affichage politique :

« Nous, on devait tenir face à la logique du parapluie, et garder vaille que vaille cette ligne de conduite. [...] [Le parapluie], c'était évidemment l'interdiction, une mesure d'interdiction spécifique aux éthers de glycol. C'est ça la logique des cabinets, c'est ce que demandent les directeurs de cabinet, parce qu'ils ont peur. Le seul argument qu'on avait pour leur faire peur à notre tour, c'était de leur dire : 'Si vous interdisez aujourd'hui les éthers de glycol, sur la foi des données disponibles, qui sont fragiles, alors il y a derrière 500, 600, 700 substances pour lesquelles il va falloir faire de même, ça va créer un précédent très problématique ${ }^{21}$." "

Le ministère accepte néanmoins, à la demande du Collectif, d'organiser, le 30 avril 2002, une table ronde pour mettre en discussion les résultats de l'appel à témoignages auprès de plusieurs spécialistes reconnus des questions de santé au travail liées aux toxiques professionnels : Marcel Goldberg, épidémiologiste membre du département Santé et Travail de l'Institut de veille

20. A titre d'exemple, on retiendra que la création de " tableaux de maladies professionnelles » reconnaissant des liens entre certains toxiques présents sur les lieux de travail et santé des salariés n'a, hormis la période contemporaine, été possible qu'aux moments où ce rapport de force est, temporairement, devenu plus favorable aux organisations syndicales, à la Libération et au début des années 1970 (Platel, 2009).

21. Entretien de l'auteur avec une fonctionnaire retraitée à la direction des Relations du travail, janvier 2006. 
sanitaire, ou encore Sylvaine Cordier et Luc Multigner, épidémiologistes de l'Inserm ayant participé à l'expertise collective sur les éthers de glycol. Dans l'espoir de légitimer auprès de ces spécialistes les résultats de l'appel à témoignages, le médecin représentant la Ligue contre le cancer au sein du Collectif demande à l'un de ses collègues biostatisticien à l'Institut Curie d'analyser les huit cas de cancers du testicule pour déterminer s'ils sont anormalement nombreux. En émettant des hypothèses hautes et basses sur la pyramide des âges des salariés d'IBM, ce biostatisticien parvient à la conclusion que les huit cas de cancer ne constituent pas un nombre anormalement élevé, et qu'ils se situent dans une fourchette de 6 à 12,4 cas attendus. Invité par Marcel Goldberg à présenter ses résultats lors de la table ronde du 30 avril, il fait l'objet de violentes critiques. André Cicolella lui reproche d'avoir utilisé les données recueillies lors de l'appel à témoignages sans l'accord préalable du Collectif. Suite à la table ronde, il interpelle également le représentant de la Ligue contre le cancer, qui quitte alors le Collectif.

Le fiasco de la table ronde marque un désaccord durable entre le Collectif et ses potentiels alliés dans le champ de la santé au travail, privant le mouvement social d'indispensables relais pour faire reconnaitre la dangerosité des éthers de glycol. Il a également des répercussions au sein du Collectif : le violente confrontation entre «leur » expert et les experts dominants du champ de la santé au travail amène les membres du Collectif à prendre leurs distances avec les positions défendues par André Cicolella et avec l'hypothèse de la cancérogénécité des éthers de glycol, indispensable pour rendre exploitables les résultats de l'appel à témoignages. André Cicolella se retrouve alors isolé sur ce sujet au sein du Collectif, son travail étiologique pour relier les résultats de l'appel à témoignages à la dangerosité des éthers de glycol suscitant de moins en moins l'adhésion des autre participants de la mobilisation :

«Médecin FMF - Moi je voudrais quand même attirer l'attention sur le fait que M. [membre du groupe d'expertise collective de l'Inserm sur les éthers de glycol et participant à la table ronde d'auril 2002], c'est le prototype de l'expert neutre qui nous a coulés, puisque il avait dit à l'époque de la table ronde que nous étions des "activistes militants partisans sans arguments scientifiques". Et il est vrai qu'à l'époque, nous étions allés un peu vite concernant la cancérogénèse. [...]

A. Cicolella - On est peut-être allé trop vite, mais on voit de mieux en mieux que nos arguments de l'époque se tenaient, puisque une étude conduite par nos adversaires prouve qu'il y a une modification qualitative du sperme, atteignant les cellules souches, ce qui constitue une altération génétique qui n’est pas sans lien avec les mécanismes de cancérogénèse. [...]

Médecin FMF - André, je dois rappeler que cette table ronde d'il y a deux ans et demi, elle a permis à nos adversaires de nous contrer parce qu'on a été maladroits, et qu'on s'est retrouvés isolés. 
A. Cicolella - Oui, c'est vrai, ils nous ont déstabilisés.

Médecin FMF - Et moi je crois qu'on n'a pas besoin d'avoir Goldberg, par exemple, contre nous ${ }^{22}$. »

Faute de données probantes, les organisations de travailleurs ont alors apporté un soutien de moins en moins ferme à l'action du Collectif éthers de glycol. Privilégiant le maintien de liens de confiance avec l'administration et les experts les plus légitimes du champ de la santé au travail, elles ont progressivement renoncé à relancer de nouvelles tentatives d'épidémiologie populaire permettant éventuellement de détecter d'autres agrégats de victimes potentielles des éthers de glycol (Jouzel, 2009). Cette défection explique pour partie le retour durable à l'anonymat de ces substances, qui sont aujourd'hui gérées selon un mode relativement routinier par l'administration de la santé au travail. En l'absence de soutien de leurs partenaires institutionnels habituels dans les luttes qui les opposent au patronat au sujet de la reconnaissance des maladies professionnelles, les organisations syndicales n'ont pu durablement maintenir un travail étiologique leur permettant de rendre visibles les dangers des éthers de glycol pour la santé des travailleurs.

\section{Conclusion}

La carrière publique des éthers de glycol comme enjeu de santé au travail aux Etats-Unis et en France appelle plusieurs séries de commentaires conclusifs. En premier lieu, il convient d'insister sur la surprenante divergence des trajectoires politiques que ces substances ont empruntées de part et d'autre de l'Atlantique. Aux Etats-Unis, elles sont rapidement apparues aux yeux des avocats et des travailleurs cherchant à faire reconnaittre les maladies des salles blanches comme un faux enjeu, construit de toutes pièces par les industriels pour masquer l'étendue des connexions causales entre les substances nécessaires pour fabriquer les puces et la santé de leurs salariés. En France, à l'inverse, l'histoire que nous avons racontée est celle d'une focalisation des mobilisations de travailleurs sur le cas particuliers des éthers de glycol : tout le travail étiologique des organisations engagées dans cette action collective a consisté à produire des données légitimant la thèse de la dangerosité spécifique de ces substances.

Cet article doit attirer l'attention sur le fait que les opérations que les organisations de travailleurs mènent afin de rendre visibles des liens incertains entre santé et toxiques professionnels ne sont pas le fruit d'une pure volonté de savoir. Le travail étiologique réalisé pour montrer les dangers de certaines substances auxquelles sont exposés les salariés est en grande partie modelé par le cadre juridique et politique dans lequel il s'inscrit. Aux EtatsUnis, les ressources et les contraintes propres au droit de la reconnaissance

22. Compte rendu d'observation de la réunion du Collectif éthers de Glycol du 15 décembre 2004. 
des maladies professionnelles ont poussé les avocats californiens à mettre en avant l'hypothèse d'une étiologie des maladies de leurs clients liée à l'ensemble des substances présentes dans les salles blanches, et non à des molécules particulières. La possibilité d'imputer la grande incertitude entourant cette hypothèse à une volonté délibérée de la part d'IBM de masquer la dangerosité du travail en salle blanche a en effet constitué un puissant atout pour obtenir l'ouverture d'un procès contre cet industriel. En France, le travail étiologique conduit par le Collectif éthers de glycol pour rendre visibles les effets de ces substances sur la santé des travailleurs a quant à lui été profondément contraint par la nécessité, pour les acteurs engagés dans cette mobilisation, de maintenir des liens de confiance avec les autorités politiques et scientifiques les plus légitimes dans le champ de la santé au travail. Renforcer l'hypothèse d'un lien de causalité entre éthers de glycol et santé des travailleurs a d'abord été vu par les organisations de travailleurs comme une ressource, leur permettant de dénoncer un problème de santé au travail techniquement soluble par la substitution des substances de la série E par celles de la série P. Mais à mesure que ce travail étiologique a suscité la défiance des acteurs administratifs et académiques dont l'appui leur est nécessaire pour peser sur l'orientation des politiques de prévention des risques professionnels, les organisations de travailleurs ont préféré y renoncer, contribuant ainsi à un retour des éthers de glycol vers un certain anonymat.

Ce dernier point nous conduit, enfin, à mettre en évidence les limites des ruptures politiques introduites par la crise de l'amiante dans les luttes syndicales contre les toxiques professionnels. Si le scandale de l'amiante a contribué à libérer la parole syndicale sur ce sujet, et a incité les organisations de travailleurs à dénoncer les lacunes du contrôle des substances toxiques sur les lieux de travail, obtenir la reconnaissance des maladies des travailleurs exposés aux substances nocives reste aujourd'hui très difficile. L'histoire des éthers de glycol permet, en creux, de rendre compte du statut d'exception de la mobilisation contre l'amiante, dont le relatif succès doit beaucoup au faible degré d'incertitude entourant les liens entre cette fibre et les maladies des travailleurs. En situation de plus grande incertitude, lorsque la toxicité d'un produit n'a été prouvée que sur l'animal, les organisations syndicales restent fortement dépendantes de la bonne volonté de leurs partenaires dans les négociations sur les politiques de santé au travail. Pour le dire autrement, en dépit de ses nombreux effets sociaux, la crise de l'amiante est loin d'avoir totalement marginalisé les formes de gestion négociée des enjeux de santé au travail, auxquelles le travail étiologique nécessaire pour faire reconnaitre les liens entre toxiques professionnels et santé des travailleurs reste fortement soumis. 


\section{Références bibliographiques}

Akrich M., Barthe Y., Rémy C. (2010), « Les enquêtes profanes et la dynamique des controverses en santé environnementale ", in Akrich M., Barthe Y., Rémy C. (dir.), Sur la piste environnementale. Menaces sanitaires et mobilisations profanes, Paris, Presses des Mines, p. 7-52.

Ashford N.A., Caldart C.C. (1996), Technology, Law, and the Working Environment, Revised Edition, Washington DC, Island Press.

Brown P. (1987), « Popular Epidemiology: Community Response to Toxic WasteInduced Disease in Woburn, Massachusetts ", Science, Technology, and Human Values, vol. 12, n 3-4, p. 78-85.

Calvez M. (2009), « Les signalements profanes de clusters de cancers. Epidémiologie populaire et expertise en santé environnementale », Sciences sociales et santé, vol. 27, $\mathrm{n}^{\circ} 2$, p. 79-106.

Chateauraynaud F., Torny D. (1999), Les sombres précurseurs. Une sociologie pragmatique de l'alerte et du risque, Paris, Editions de l'EHESS.

Courtet C., Gollac M. (2012), Risques du travail, la santé négociée, Paris, La Découverte.

Crespin R. (2006), « Drogues et sécurité routière. Changement politique ou nouvel usage des instruments ? ", Revue française de science politique, vol. 56, $n^{\circ} 5$, p. $813-836$.

Déplaude M.-O. (2003), " Codifier les maladies professionnelles. Les usages conflictuels de l'expertise médicale », Revue française de science politique, vol. 53, $n^{\circ} 5$, p. 707-735.

Devinck J.-C. (2008), « Le mouvement ouvrier et les maladies professionnelles », Cahiers Risques collectifs et situations de crise, $n^{\circ}$ 9, p. 33-44.

Gordon B. (1993), « Ouvrières et maladies professionnelles sous la IIIe République. La victoire des allumetiers français sur la nécrose phosphorée de la mâchoire ", Le mouvement social, $\mathrm{n}^{\circ} 164$, p. 77-94.

Henry E. (2003), « Du silence au scandale. Des difficultés des médias d'information à se saisir de la question de l'amiante ", Réseaux, n 122, p. 237-272.

Henry E. (2007), Amiante, un scandale improbable. Sociologie d'un problème public, Rennes, PUR.

Henry E. (2011), « Nouvelles dynamiques de savoirs et permanence des rapports de pouvoir. L'impact - limité - des transformations - importantes - de l'expertise en santé au travail », Revue française de science politique, vol. 61, $\mathrm{n}^{\circ} 4$, p. $707-726$.

Jasanoff S. (1995), Science at the Bar : Law, Science, and Technology in America, Cambridge, Harvard University Press.

Jouzel J.-N. (2007), « Fausse alerte ? Le destin singulier des éthers de glycol dans l'univers de la santé professionnelle en France », Politix, vol. 20, $\mathrm{n}^{\circ} 79$, p. $175-193$.

Jouzel J.-N. (2009), « Encombrantes victimes. Pourquoi les maladies professionnelles restent-elles socialement invisibles en France ? ", Sociologie du travail, vol. $51, \mathrm{n}^{\circ} 3$, p. 402-418.

Jouzel J.-N. (2013), Des toxiques invisibles. Sociologie d'une affaire sanitaire oubliée, Paris, Editions de l'EHESS. 
Markowitz G.E., Rosner D. (2003), Deceit and Denial: The Deadly Politics of Industrial Pollution, Berkeley, University of California Press.

Oreskes N., Conway E.M. (2010), Merchants of Doubt: How a Handful of Scientists Obscured the Truth on Issues from Tobacco Smoke to Global Warming, London, Bloomsbury Press.

Pitti L. (2010), « Experts "bruts" et médecins critiques. Ou comment la mise en débats des savoirs médicaux a modifié la définition du saturnisme en France durant les années 1970 », Politix, n 91, p. 103-132.

Platel S. (2009), "La reconnaissance des cancers professionnels. Entre tableaux et CRRMP, une historique prudence à indemniser... ", Mouvements, $n^{\circ} 58$, p. 46-55.

Proctor R.N. (2012), Golden Holocaust: Origins of the Cigarette Catastrophe and the Case for Abolition, Berkeley, University of California Press.

Rosental P.-A., Omnès C. (2009), « L'histoire des maladies professionnelles, au fondement des politiques de "santé au travail" ", Revue d'histoire moderne et contemporaine, $\mathrm{n}^{\circ}$ 56-1, p. 5-11.

Thébaud-Mony A. (2007), Travailler peut nuire gravement à votre santé. Soustraitance des risques, mise en danger d'autrui, atteinte à la dignité, violences physiques et morales, cancers professionnels, Paris, La Découverte.

Wynne B. (1992), « Misunderstood Misunderstanding: Social Identities and Public Uptake of Science », Public Understanding of Science, vol. 1, $\mathrm{n}^{\circ} 3$, July, p. 281-304. 